Pacific Journal of Mathematics

SINGULAR PERTURBATION OF LINEAR PARTIAL
DIFFERENTIAL EQUATION WITH CONSTANT COEFFICIENTS 


\title{
SINGULAR PERTURBATION OF LINEAR PARTIAL DIFFERENTIAL EQUATION WITH CONSTANT COEFFICIENTS
}

\author{
Hussain S. NuR
}

\begin{abstract}
Let $P_{j}(z, \varepsilon)$ be a polynomial in $z$ and $\varepsilon$ with complex coefficients, where $z$ is in $E^{m}$ and $\varepsilon>0$ is a small parameter. Let $L_{\varepsilon}=\sum_{j=0}^{l} P_{l-j}\left(\delta_{x}, \varepsilon\right)\left(\delta_{t}\right)^{\jmath}$ be a polynomial in $\delta_{t}, \delta_{x}$ and $\varepsilon$, which is not divisible by the square of a similar nonconstant polynomial. We shall assume that $P_{0}(z, \varepsilon)=\varepsilon$ and $P_{1}(z)$ is independent of $\varepsilon$.

In this paper we shall show that under certain conditions the solution $u_{\varepsilon}(t, x)$ of $L_{\varepsilon}(u)=f_{\varepsilon}(t, x)$ converges to the solution $u_{0}(t, x)$ of $L_{0}(u)=f_{0}(t, x)$.
\end{abstract}

Let $(t, x)=\left(t, x_{1}, x_{2}, \cdots, x_{m}\right)$ be a point in $R \times E^{m}$ where $0 \leqq t \leqq T$, and $x$ in $E^{m}$, and $E^{m}$ denotes an $m$-dimensional Euclidean space. Let also $C_{0}^{\infty}$ be the set of all infinitely times continuously differentiable complex valued functions on $E^{m}$ with compact support. For any $u$ in $C_{0}^{\infty}$, let the norm $\|u\|_{p}$ be defined for any integer $p<0$ as follows:

$$
\int_{E^{m}} \sum_{|\varphi| \leqq p}\left|\partial_{x_{1}}^{\omega_{1}} \cdots \partial_{x_{m}}^{\varphi_{m}}\right|^{2} d x=\|u\|_{p}^{2} \quad\left(|\varphi|=\varphi_{1}+\cdots+\varphi_{m}\right) .
$$

It is easy to see that the space $C_{0}^{\infty}$ with the norm (1) gives a Hilbert space, which we shall call an $H_{p}$-space. We may also notice that $H_{p} \supset H_{q}$ and $\|u\|_{p} \leqq\|u\|_{q}$ if $p<q$. If for each $\varphi$ in $H_{p}$ we denote by $\hat{\varphi}$ the Fourier transform of $\varphi$

$$
\widehat{\varphi}(z)=\left[1 /(2 \pi)^{m / 2}\right] \int_{E^{m}} \exp (-i x . z) \varphi(x) d x
$$

where,

$$
x, z=\sum_{1}^{m} x_{i} z_{i}
$$

then the norm defined in (1) will be equivalent to the norm

$$
\|\varphi\|_{p}^{2}=\int_{E m}\left|\left(1+|z|^{2}\right)^{p / 2} \hat{\varphi}(z)\right|^{2} d z=\|\hat{\varphi}\|_{p}^{2} .
$$

Notice that $H_{p}$ with respect to the norm defined in (2) is the set of all complex valued measurable functions such that $\|\varphi\|_{p}<\infty$.

Let $D^{k}$ be any differential operator with respect to $x$ with constant coefficients of order $k \leqq p$. Then $D^{k}$ is a bounded linear operator which maps $H_{p}$ into $H_{p-k}$. 
DEFINITION 1. Let $\varphi(t)$ be a variable element of $H_{p}$ depending on a real parameter $t$ in a finite interval $J=[0, T]$. We say that $\varphi(t)$ is $H_{p}$-continuous in $t$ in $J$, if the mapping $t$ in $J \rightarrow \varphi(t)$ in $H_{p}$ is continuous; That is, $t \rightarrow t_{0}$ in the interval $J$ implies $\varphi(t) \rightarrow \varphi\left(t_{0}\right)$ in $H_{p}$. We also maintain that $\varphi(t)$ is $H_{p}$-differentiable at $t=t_{0}$, if there exist a function $g(t)$ in $H_{p}$ such that

$$
\left(t-t_{0}\right)^{-1}\left[\varphi(t)-\varphi\left(t_{0}\right)\right] \rightarrow g\left(t_{0}\right)
$$

in $H_{p}$ as $t \rightarrow t_{0}$, then we denote $g(t)$ by $\varphi^{\prime}(t)=(d / d t) \varphi(t)$.

If $D^{k}$ is a differential operator in $x$ in $E^{m}$ with constant coefficients of order $k$ and $\varphi(t)$ is $H_{p}$-continuous in $t$, then $D^{k} \varphi(t)$ is $H_{p-k}$ continuous, and if $\varphi(t)$ is $H_{p}$-differentiable in $t$ then $D^{k} \varphi(t)$ is $H_{p-k}$ differentiable in $t$ and

$$
(d / d t)\left[D^{k} \varphi(t)\right]=D^{k}[(d / d t) \varphi(t)] .
$$

Mr. Nagumo in [1] considered a system of linear partial differential equations for an $r$-vector function with parameter $\varepsilon>0$,

$$
L_{\varepsilon}(u)=\sum_{j=0}^{l} P_{j}\left(\partial_{x}, \varepsilon\right)\left(\partial_{t}\right)^{j} u=f_{\varepsilon}(t, x)
$$

where $P_{j}(z, \varepsilon)$ are $r \times r$ matrices of polynomials in $(z, \varepsilon)$ with constant coefficients and $P_{l}$ is free from $\partial_{x}$ such that $\operatorname{det}\left[P_{l}(\varepsilon)\right] \neq 0$ for $\varepsilon=0$.

Here we are concerned with the case of one equation for one complex valued function $u(t, x)$ containing the parameter $\varepsilon>0$

$$
L_{\varepsilon}(u)=\sum_{j=0}^{l} P_{j}\left(\partial_{x}, \varepsilon\right)\left(\partial_{t}\right)^{j} u=f_{\mathbf{\imath}}(t, x)
$$

with the following assumptions:

(0) $L_{\varepsilon}(\sigma)$ be a polynomial in $\sigma, \partial x$ and $\varepsilon$ which is not divisible by the square of a similar nonconstant polynomial for $0 \leqq \varepsilon \leqq \varepsilon_{0}$ and $f_{\varepsilon}(t, x)$ is $H_{p}$-continuous. $P_{j}(z, \varepsilon)$ are polynomials in $(z, \varepsilon)=\left(z_{1}, \cdots, z_{m}, \varepsilon\right)$ with constant coefficients such that $P_{l}(\varepsilon) \equiv \varepsilon$ and $P_{l-1}(z)$ is independent of $\varepsilon$.

System (4) is certainly a special case of system (3). Restricting ourselves to this special case, we will prove a stability theorem somewhat different from that of $\mathrm{Mr}$. Nagumo [1]. Mr. Nagumo proved the convergence of the weak solution to $u_{0}(t, x)$; where as we shall prove the convergence of the solution $u_{\varepsilon}(t, x)$ to $u_{0}(t, x)$.

Definition 2. We say that equation (4) is an $H_{p}$-stable equation for $\varepsilon \rightarrow 0$ in $0 \leqq t \leqq T$ with respect to a particular solution $u_{0}(t, x)$ of (4) for $\varepsilon=0$ if and only if $u_{\varepsilon}(t) \rightarrow u_{0}(t)$ in $H_{p}$ for $0 \leqq t \leqq T$ provided that 


$$
f_{\varepsilon}(t, x) \rightarrow f_{0}(t, x)
$$

in $H_{p}$ for $0 \leqq t \leqq T$ and $u_{\varepsilon}(t, x)$ is a solution of the partial differential equation (4) such that

(i ) $\partial_{t}^{j-1} u_{\varepsilon}(0) \rightarrow \partial_{t}^{j-1} u_{0}(0)$ in $H_{p} \quad(j=1, \cdots, l-1)$.

(ii) There exists a function $F(x)$ in $H_{p}$ such that

$$
\left|\partial_{t}^{l-1} \widehat{u}_{\varepsilon}(0, z)\right| \leqq|\hat{F}(z)| \text { for all small } \varepsilon>0 \text {. }
$$

As in [1] we associate the partial differential equation (4) with the ordinary differential equation

$$
\sum_{j=0}^{l} P_{j}(i z, \varepsilon)(d / d t)^{j} y=0 .
$$

Let $Y_{j}(t, z, \varepsilon)$ be the solution of the ordinary differential equation (7) with the initial conditions

$$
\partial_{t}^{k-1} Y_{j}(0, z, \varepsilon)=\delta_{j k} \quad\left(\delta_{j k} \text { is the Kronecker delta }\right) .
$$

We state here a well known result

Theorem 1. Let $P(z)$ be a polynomial in $z\left(z\right.$ in $\left.E^{m}\right)$ with complex coefficients. If $S=(z$ such that $P(z)=0)$ then $S$ is measurable and has $E^{m}$ measure zero unless $P(z)$ is identically zero.

The proof is simple. One approach is to use mathematical induction on $m$ and Fubini's theorem.

COROLLARY. There exist an $\varepsilon_{0}>0$ such that for each $\varepsilon$ in $0 \leqq \varepsilon \leqq \varepsilon_{0}$ then assumption (0) implies that the polynomial equation

$$
\sigma^{l}+P_{l-1} \sigma^{l-1}+\cdots+P_{0}(i z, \varepsilon)=0
$$

has distinct roots except for $z$ belonging to a set of $E^{m}$ measure zero.

Proof. Notice that the assumption (0) implies that $D(z, \varepsilon)$ the discriminant of equation (9) is not identically zero. $D(z, \varepsilon)$ is a polynomial of $\left(z_{1}, \cdots, z_{m}, \varepsilon\right)$. Let us write $D(z, \varepsilon)$ as a product of irreducible polynomials in $z$ and $\varepsilon$ over the field of complex coefficients. If one or several of the factors do not depend on $z$ explicitly, then they are polynomials in $\varepsilon$; in fact they are linear. All of these have at most finitely many positive zeros, say $\varepsilon_{1}, \cdots, \varepsilon_{n}$. Let $\varepsilon_{0}=\min \left(\varepsilon_{1}, \cdots, \varepsilon_{n}\right)$; then for $\varepsilon \varepsilon_{0}$ we can write $D(z, \varepsilon)$ as a product of irreducible polynomials in $z$ and $\varepsilon$ none of which vanishes identically. Now by Theorem 1 the zeros of such polynomials for each $\varepsilon$ are set of $E^{m}$ measure zero.

Let $Y_{i}(t, z, \varepsilon)(i=1,2, \cdots, l)$ be the solution of the ordinary dif- 
ferential equation (7) with the initial conditions (8). If $\sigma_{1}, \cdots, \sigma_{l}$ are the distinct roots of equation (9) then we can write

$$
Y_{i}(t, z, \varepsilon)=\sum_{j=1}^{l} \alpha_{j}^{i} \exp \left(\sigma_{j} t\right) \text {. }
$$

Here $\alpha_{\jmath}^{i}$ are constants to be computed by using the initial conditions (8). Let $V\left(\sigma_{1}, \cdots, \sigma_{l}\right)$ be the Vandermond determinant of $\sigma_{1}, \cdots, \sigma_{l}$, i.e., $V\left(\sigma_{1}, \cdots, \sigma_{l}\right)=\pi_{q>p}\left(\sigma_{q}-\sigma_{p}\right)$. Denote by $V_{i}^{j}$ the determinant obtained from $V$ by cancelling the $i$-th column and the $j$-th row.

THEOREM 2. $V_{i}^{j}=\pi_{q p, q \neq j \neq p}\left(\sigma_{q}-\sigma_{p}\right) E_{j, i}$ where $E_{j, i}$ is the coefficient of the $i$-th power of $\sigma_{j}$ in the expression

$$
\left(\sigma_{1}-\sigma_{j}\right) \cdots\left(\sigma_{j-1}-\sigma_{j}\right)\left(\sigma_{j+1}-\sigma_{j}\right) \cdots\left(\sigma-\sigma_{j}\right)(-1)^{j} .
$$

The proof is simple. Just write $V\left(\sigma_{1}, \cdots, \sigma_{l}\right)$ in two ways; first as a polynomial in $\sigma_{j}$, and second as a product of linear terms then equate the coefficients of $\sigma_{j}$ in the two expressions.

Then initial conditions (8) and further use of Vandermond determinant give the following result

$$
Y_{i}(t, z, \varepsilon)=(-1)^{i-1}\left[\sum_{j=1}^{l} E_{j,(i-1)} \exp \left(\sigma_{j}\right) / A_{j}\right]
$$

where,

$$
A_{j}=\left(\sigma_{l}-\sigma_{j}\right) \cdots\left(\sigma_{j-1}-\sigma_{j}\right)\left(\sigma_{j+1}-\sigma_{j}\right) \cdots\left(\sigma_{1}-\sigma_{j}\right) .
$$

Since the preceding result can be computed easily, we shall omit the details.

Theorem 3. If $\sigma_{1}, \cdots, \sigma_{l}$ are the roots of equation (9) and $Y_{1}$, $Y_{2}, \cdots, Y_{l}$ are the solutions of the ordinary differential equation (7) with the initial conditions (8). Then for each $\sigma_{j} \neq 0(j=1, \cdots, l)$ we have

$$
\sigma_{j}^{l-1} \sum_{i=1}^{l}\left(Y_{i} / \sigma_{j}^{l-1}\right)=\exp \left(\sigma_{j} t\right)
$$

Proof. The initial conditions (8) shows that the identity (13) is valid for $t=0$. Furthermore take the 1st, 2 nd, $\cdots,(l-1)$-th derivatives of both sides of the identity with respect to $t$ and each time apply the initial conditions (8) we get the validity of the identity for $t=0$. Since the right side of equation (13) is a solution of the ordinary differential equation (7) therefore the identity (13) is valid for all $t$ in $0 \leqq t \leqq T$. 
THEOREM 4. For each fixed $z$ in $E^{m}$ consider $Y_{j}(t, z, \varepsilon)$ a function of $t$ and $\varepsilon$ only and assume that there exists a number $M_{j}(z)(j=1, \cdots, l)$ independent of both $t$ and $\varepsilon$ such that

$$
\left|Y_{j}(t, z, \varepsilon)\right| \leqq M_{j}(z)
$$

for $0<\varepsilon \leqq \varepsilon_{0}$ and $0 \leqq t \leqq T$. Then the roots of equation (9) have for each $z$ in $E^{m}$ a real part bounded from above as $\varepsilon \rightarrow 0$.

Proof. Let $z_{0}$ be a fixed point in $E^{m}$. Let $\mu=\varepsilon \sigma$ when $\sigma$ is a root of equation (9) then, equation (9) becomes

$$
\mu^{l}+P_{l-1}\left(i z_{0}\right) \mu^{l-1}+\varepsilon P_{2}(i z, \varepsilon) \mu^{l-2}+\cdots+\varepsilon^{l-1} P_{0}(i z, \varepsilon)=0 .
$$

Now assume first that $P_{l-1}\left(i z_{0}\right) \neq 0$ then for $\varepsilon=0$ equation (14) becomes

$$
\mu^{l}+P_{l-1}\left(i z_{0}\right) \mu^{l-1}=0=\mu^{l-1}\left(\mu+P_{l-1}\left(i z_{0}\right)\right) .
$$

Here we have one simple root $\mu_{1}=-P_{l-1}\left(i z_{0}\right)$, if we call this root $\mu_{1}(0)$ then we can write

$$
\mu_{1}(\varepsilon)=\mu_{1}(0)+\sum_{1}^{\infty} q_{1 i} \varepsilon^{i} \text {, so } \mu_{1}(\varepsilon) \rightarrow \mu_{1}(0) \text { as } \varepsilon \rightarrow 0 .
$$

Therefore we can write the simple root $\sigma_{1}\left(z_{0}, \varepsilon\right)$ of equation (14) as follows,

$$
\sigma_{1}=(1 / \varepsilon) \mu_{1}(\varepsilon)=\left(-P_{l-1}\left(i z_{0}\right) / \varepsilon\right)+\sum_{i=1}^{\infty} q_{l i}\left(z_{0}\right) \varepsilon^{i-1} .
$$

Hence Real $\sigma_{1}\left(z_{0}, \varepsilon\right)=-$ Real $P_{l-1}\left(i z_{0}\right) / \varepsilon+$ Real $q_{11}\left(z_{0}\right)+o(\varepsilon)$. Therefore if Real $P_{l-1}\left(i z_{0}\right) \geqq 0$ then obviously Real $\sigma_{1}(\varepsilon)$ is bounded from above. Now suppose that Real $P_{l-1}<0$. Then $\left|\sigma_{1}(\varepsilon)\right| \geqq \operatorname{Real}\left(\sigma_{1}(\varepsilon)\right) \rightarrow \infty$ in turn implies that for $t>0$ we get

$$
\left|\sum_{1}^{l}\left[Y_{j}\left(t, z_{0}, \varepsilon\right) / \sigma^{l-j}\right]\right| M_{j}\left(z_{0}\right)
$$

as $\varepsilon \rightarrow 0$ for some number $M_{j}\left(z_{0}\right)$ independent of $t$ and $\varepsilon$. This is so because of the hypothesis of the theorem. Now we use Theorem 3 . Then identity (13) shows for small

$$
\varepsilon>0,2\left|P_{l-1}\right| \geqq \mid-P_{l-1}\left(i z_{0}+0(\varepsilon)|\geqq(1 / 2)| P_{l-1}\left(i z_{0}\right) \mid\right.
$$

since Real $P_{l-1}\left(i z_{0}\right)<0$ and as $t>0$,

$$
\begin{aligned}
& {\left[\exp \left(t \operatorname{Re} \sigma_{1}\right) / \sigma_{1}^{l-1} \mid\right] \geqq\left[\exp \left(2_{2}^{t} \varepsilon\left|P_{l-1}\right|\right) \varepsilon^{l-1} /\left(4\left|P_{l-1}\left(i z_{0}\right)\right|\right)^{l-1}\right]} \\
& \geqq\left(t / 4^{l} l ! \varepsilon\right) \rightarrow \infty \text { as } \varepsilon \rightarrow 0
\end{aligned}
$$

utilizing only the $(l-1)$ th term of the Taylor series of the exponential function. 
But the above result contradicts the boundedness of expression (13) for small $\varepsilon>0$. Consequently Real $P_{l-1}\left(i z_{0}\right) \geqq 0$ and hence Real $\sigma_{1}(\varepsilon)$ is bounded from above as $0<\varepsilon \leqq \varepsilon_{0}$.

In order to prove the result for the remaining of the roots of equation (9) we shall give a reasoning which is incidentally does not utilize the condition $P_{l-1}\left(i z_{0}\right) \neq 0$. Equation (15) has a root $\mu(0)=0$ of multiplicity $(l-1)$. From the Puiseux series expansion we deduce that the $(l-1)$ roots $\mu(\varepsilon)$ of equation (14) other than $\mu_{1}$ will split into $r$ groups of $m_{1}, \cdots, m_{r}, 1 \leqq m_{1} \leqq \cdots \leqq m_{r}$ and $\sum_{1}^{r} m_{i}=l-1$ as follows: each root $\mu_{0 j}, \cdots, \mu_{m_{j-1} j}$ can be written as $\mu_{\eta_{j}}=\sum_{j=1}^{\infty} q_{\eta_{j i}} x^{i}$ where,

$$
x=(\varepsilon)^{1 / m} j \text { and } \eta=0,1, \cdots, m_{j-1} .
$$

Notice that the above series converges for sufficiently small $x$. We shall here and later understand by $(\varepsilon)^{1 / m} j$ the positive $m_{j}$-th root of $\varepsilon>0$. Let $\mu_{\eta j}$ be any one of the $(l-1)$ roots of equation (14) which tend to zero as $\varepsilon$ tends to zero, then we can write the corresponding roots $\sigma_{\eta}$ of equation (9) as follows

$$
\left(\mu_{\eta} / \varepsilon\right)=\sigma_{\eta_{j}}\left(x, z_{0}\right)=\left(1 / X^{m} j\left(\sum_{i=1}^{\infty} q_{\eta j} x^{i}\right)\right) .
$$

Now to simplify notations, let us drop indices $j, \eta$ of the root $\mu_{\eta_{j}}$ once we are dealing with only one root. Put $q \eta_{j i}=q_{i}$ and $m_{j}=m$. Assume that $q_{k}$ is the first nonzero coefficient in equation (16) if there is any, and $q_{s}$ is the first nonzero coefficient that has a nonzero real part, if there is any, in the expresion $\mu=\sum q_{i} x^{i}$. Evidently, if $s \geqq m$ then, Real $\sigma\left(x, z_{0}\right)=x^{s-m}$ Real $q_{s}+0(x)$, and this is bounded from above as $x \rightarrow 0$. If there is no $s$, then $\sigma\left(x, z_{0}\right) \leqq 0$. Let $s<m$, notice that $k \leqq s \leqq m$. Then we can write $\sigma$ as follows,

$$
\sigma(x)=x^{k-m}\left(q_{k}+\cdots+q_{s} x^{s-k}+q_{s+1} x^{s-k+1}+\cdots\right)
$$

and

$$
\text { Real } \sigma(x)=x^{s-m}\left(\operatorname{Real} q_{s}+0(x)\right) .
$$

If Real $q_{s}<0$ then Real $\sigma(x) \rightarrow-\infty$ as $x \rightarrow 0$ implying Real $\sigma \leqq 0$ as $\varepsilon \rightarrow 0$. Suppose finaly Real $q_{s}>0$, i.e., Real $\sigma(x) \rightarrow+\infty$ as $x \rightarrow 0$. Then for small $x>0,2\left|q_{k}\right| \geqq\left|q_{k}+0(x)\right|$ and Real $q_{s}+0(x) \geqq \operatorname{Re} q_{s} / 2$, and

$$
\begin{gathered}
\exp (t \operatorname{Re} \sigma) /|\sigma|^{l-1} \geqq \exp \left({ }_{2}^{t} \operatorname{Re} q_{s} x^{s-m}\right) / 2^{l-1}\left|q_{k}\right|^{l-1} x^{(k-m)(l-1)} \\
>\left(t^{j} / j ! 2^{j}\right) x^{j(s-m)}\left(\operatorname{Real} q_{s}\right)^{j} / 2^{l-1}\left|q_{k}\right|^{\mid l-1} x^{(k-m)(l-1)} .
\end{gathered}
$$

Here we utilize only the $(j+1)$-th term of the Taylor series of the exponential function where $j$ is the smallest integer greater than zero 
such that $(m-k)(l-1)+j(s-m)<0$. Therefore

$\exp (t$ Real $\sigma) /|\sigma|^{l-1}>t^{j}\left(\operatorname{Real} q_{s}\right)^{j} x^{(m-k)(l-1)+j(s-m)} / j ! 2^{j+l-1}\left|q_{k}\right|^{l-1} \rightarrow \infty$

as $x \rightarrow 0$ because of the assumption on $j$.

Now in order to prove that Real $\sigma$ is bounded from above we use identity (13) once more.

In order to complete the proof of the theorem we should assume that $P_{l-1}\left(i z_{0}\right)=0$. Notice that equation (14) for $P_{l-1}=0$ becomes

$$
\mu^{l}+\varepsilon P_{l-2}\left(i z_{0}, \varepsilon\right)+\cdots+\varepsilon^{l-1} P_{0}\left(i z_{0}, \varepsilon\right)=0 .
$$

This means that $\mu=0$ is a root of multiplicity $l$, not $l-1$, of equation (15). So by Puiseux series expansion we can write the roots

$$
\begin{array}{ll}
\sigma_{1 \eta_{1}}=(1 / \varepsilon)\left[\sum_{j=1}^{\infty} \beta_{1 j} \exp \left(2 \pi i \eta_{1} j / m_{1}\right) \varepsilon^{j / m_{1}}\right] & \eta_{1}=0, \cdots, m_{1}-1 \\
\vdots & \\
\sigma r \eta_{r}=(1 / \varepsilon)\left[\sum_{j=1}^{\infty} \beta_{r j} \exp \left(2 \pi i \eta_{r} j / m_{r}\right) \varepsilon^{j / m_{r}}\right] & \eta_{r}=0, \cdots, m_{r}-1 .
\end{array}
$$

Now we can carry on the same proof as before for any root. The proof of Theorem 4 is completed.

In what follows there will be for each $\varepsilon$ certain exceptional sets of $z$ of measure zero for which our conclusion do not apply. In order to be able to draw inferences as $\varepsilon \rightarrow 0, \varepsilon>0$ we wish to be able to disregard these sets. Now let the notion $\varepsilon \rightarrow 0$ be henceforth interpreted as meaning " $\varepsilon$ tends to zero through an arbitrary sequence of positive numbers." Then all of the corresponding exceptional sets will still be a countable union of sets of measure zero and accordingly has itself measure zero.

Theorem 5. Assume in equation (9) that $P_{l-1}\left(i z_{0}\right)$ does not vanish identically. Also assume that for $0 \leqq t \leqq T, 0<\varepsilon \leqq \varepsilon_{0}$, there are numbers $M(z)$ and $M_{j}(z)(j=1, \cdots, l)$ independent of both $t$ and $\varepsilon$ such that,

$$
\left|Y_{j}(t, z, \varepsilon)\right| \leqq M_{j}(z) \text { for } 0<\varepsilon \leqq \varepsilon_{0} .
$$

Then for almost all $z$ in $E^{m}$ we have

(i) $\left|Y_{l}(t, z, \varepsilon)\right| \rightarrow 0$ as $\varepsilon \rightarrow 0$ and for $0 \leqq t \leqq T$.

(ii) $\left|(1 / \varepsilon) Y_{l}(t, z, \varepsilon)\right| \leqq M(z)$ for $0 \leqq t \leqq T$ and as $\varepsilon \rightarrow 0$.

Proof. Here we use Theorem 1 in order to be able to assume $P_{l-1}\left(i z_{0}\right) \neq 0$. By the corollary of Theorem 1, equation (9) has for fixed small $\varepsilon$ and for almost all $z$ in $E^{m}$ distinct roots. By letting $\mu=\varepsilon \sigma$ the equation (9) becomes as we have seen before 


$$
\mu^{l}+P_{l-1}\left(i z_{0}\right) \mu^{l-1}+\varepsilon P_{l-1}\left(i z_{0}, \varepsilon\right) \mu^{l-2}+\cdots+\varepsilon^{l-1} P_{0}\left(i z_{0}, \varepsilon\right)=0 .
$$

So for $\varepsilon=0$ the above equation will be,

$$
\mu^{l-1}\left(\mu+P_{l-1}\left(i z_{0}\right)\right)=0 .
$$

Therefore by Puiseux series expansion we can write the roots of equation (9) as follows,

$$
\sigma_{1}=(1 / \varepsilon)\left[-P_{l-1}\left(i z_{0}\right)+0(\varepsilon)\right]
$$

$m_{1}$ roots as follows,

$$
\sigma_{1+\eta_{1}}=(1 / \varepsilon)\left[\sum_{j=1}^{\infty} \beta_{1 j} \varepsilon^{j / m_{1}} \exp \left(2 \pi i \eta_{1} j / m_{1}\right)\right] \quad n_{1}=1, \cdots, m_{1}
$$

and $m_{r}$ roots as follows,

$$
\sigma_{-m_{r}+\eta_{r}}=(1 / \varepsilon)\left[\sum_{j=1}^{\infty} \beta_{r j} \exp \left(2 \pi i \eta_{r} j / m_{r}\right) \varepsilon^{j / m_{r}}\right] \quad \eta_{r}=1, \cdots, m_{r} .
$$

Provided that $l=1+m_{1}+\cdots+m_{r}$ where $1 \leqq m_{1} \leqq \cdots \leqq m_{r}$. Let us write $\gamma=\left[\sigma_{1}, \cdots, \sigma_{l}\right]$. Notice that each $\sigma$ in $\gamma$ is a regular function in $\varepsilon$ and hence the difference between any two of them is also regular in $\varepsilon$. If $\sigma_{1}=(1 / \varepsilon)\left[-P_{l-1}\left(i z_{0}\right)+0(\varepsilon)\right]$ then for any $j, 1<j \leqq l$ we have, since $P_{l-1}\left(i z_{0}\right) \neq 0$,

$$
\left|\sigma_{j}-\sigma_{1}\right|=\left|(1 / \varepsilon)\left[-P_{l-1}\left(i z_{0}\right)+0(\varepsilon)-0\left(\varepsilon^{R}\right)\right]\right| \rightarrow \infty \text { as } \varepsilon \rightarrow 0 .
$$

For $\sigma_{j}=(1 / \varepsilon)\left[0\left(\varepsilon^{R}\right)\right], R \quad 0$ a rational number.

Notice that for each $i=1, \cdots, l, i \neq j$,

$$
\left|\sigma_{j}-\sigma_{i}\right|
$$

either; (1) goes to zero as $\varepsilon$ tends to zero; (2) tends to some fixed number greater than zero or (3) goes to infinity as $\varepsilon$ tends to zero. For any arbitrary $\sigma$ in $\gamma$ collect those, and only those, elements $\sigma^{\prime}$ of $\gamma$ such that $\left|\sigma^{\prime}-\sigma\right| \rightarrow 0$ as $\varepsilon \rightarrow 0$. Then $\gamma$ will split into disjoint subsets, namely:

$$
\gamma=\gamma_{1} U \gamma_{2} \cdots U \gamma_{r} \text { and } \gamma_{j} \cap \gamma_{k}=\varnothing \text { for } j \neq k,
$$

which incidentally do not necessarily coincide with our previous grouping of the $\sigma^{\prime}$ s. According to this decomposition of $\gamma$ and using identity (10) we can write

$$
\left|Y_{l}(t, z, \varepsilon)\right|=\left|\sum_{\sigma_{j} i \gamma_{1}}\left[\exp \left(t \sigma_{j}\right) / A_{j}\right]+\cdots+\sum_{\sigma_{j} i n \gamma_{r}}\left[\exp \left(t \sigma_{j}\right) / A_{j}\right]\right| .
$$


LEMMA 1. In the above expression each summation tends to zero in absolute value as $\varepsilon$ tends to zero.

Proof. Denoting by $\sigma_{1}^{\prime}, \cdots, \sigma_{n}^{\prime}$ the elements of $\gamma_{1}$. Then,

$$
\begin{gathered}
\sum_{\sigma_{j}^{\prime} i n \gamma_{1}}\left[\exp \left(\sigma_{j}^{\prime} t\right) / A_{j}\right]=\sum_{\sum_{j}^{\prime} i n \gamma_{1}}\left[\exp \left(\sigma_{j}^{\prime} t\right) / \varphi\right] \\
\varphi=\prod_{\sigma_{k} i n \gamma_{-} \gamma_{1}}\left(\sigma_{k}-\sigma_{\jmath}^{\prime}\right) \prod_{\substack{\sigma_{k} i n \gamma_{1} \\
k \neq j}}\left(\sigma_{k}^{\prime}-\sigma_{\jmath}^{\prime}\right) .
\end{gathered}
$$

Let $F\left(\sigma_{\jmath}^{\prime}\right)=\exp \left(t \sigma_{j}^{\prime}\right) / \Pi_{\sigma_{k} i n \gamma-\gamma_{1}}\left(\sigma_{k}-\sigma_{j}^{\prime}\right)$ and $F\left(\sigma_{j}^{\prime}\right)=\alpha(\varepsilon)$. Now if $\gamma_{1}$ contains the root $\sigma_{1}=(1 / \varepsilon)\left[-P_{l-1}\left(i z_{0}\right)+0(\varepsilon)\right]$ then it will contain only $\sigma_{1}$. Since $P_{1}\left(i z_{0}\right) \neq 0$ it is easily shown that,

$$
\exp \left(t \sigma_{1}\right) / A_{1} \rightarrow 0 \text { as } \varepsilon \rightarrow 0 \text {. }
$$

Now suppose that $\gamma_{1}$ does not contain $\sigma_{1}$. Then $\prod_{\sigma_{k} i n \gamma-\gamma_{1}}\left(\sigma_{k}-\sigma_{j}^{\prime}\right)$ will have a factor $\left(\sigma_{1}-\sigma_{j}^{\prime}\right)=0(\varepsilon)$ and hence tends to infinity as $\varepsilon$ tends to zero while no factor of $\prod_{\left.\sigma_{k} i n\right\}-\gamma_{1}}\left(\sigma_{k}-\sigma_{\jmath}^{\prime}\right)$ tends to zero as $\varepsilon$ tends to zero. Therefore,

$$
\prod_{\sigma_{k} i n \gamma-\gamma_{1}}\left(\sigma_{k}-\sigma_{j}^{\prime}\right) \rightarrow \infty \text { as } \varepsilon \rightarrow 0 .
$$

This in turn implies that $\alpha(\varepsilon)$ tends to zero as $\varepsilon$ tends to zero. Let $\left.\alpha=\min _{\text {(limit }}{ }_{\varepsilon \rightarrow 0}\left|\sigma_{k}-\sigma_{\jmath}^{\prime}\right|\right)$. The minimum is taken over all $\sigma_{k}$ in $\gamma-\gamma_{1}$. Notice that by the definition of $\gamma_{1}, \delta>0$. Now chose a circle $C$ of radius $\delta / 2$ about one of the points $\sigma_{1}^{\prime}, \cdots, \sigma_{n}^{\prime}$ of $\gamma_{1}$. Then for sufficiently small $\varepsilon>0, C$ will contain those, and only those, $\sigma_{j}^{\prime}$ which belong to $\gamma_{1}$. We may likewise assume that for any point $w$ on the circumference of $C$, that $\left|w-\sigma_{j}^{\prime}\right|>\delta / 4,(j=1, \cdots, n)$. Let

$$
I=(1 / 2 \pi i) \int_{C} F(\eta) d \eta / \prod_{\tilde{o}_{k}^{\prime} i n \gamma_{1}}\left(\sigma_{k}^{\prime}-\eta\right)
$$

then,

$$
I<4^{n} \alpha(\varepsilon) / \delta^{n} \rightarrow 0 \text { as } \varepsilon \rightarrow 0 .
$$

On the other hand, $I$ equal the absolute value of the sum of the residues of the integrand at $\sigma_{1}^{\prime}, \cdots, \sigma_{n}^{\prime}$. Notice that for each $\sigma_{j}^{\prime}$ the residue of $I$ at $\sigma_{j}^{\prime}$ is $F\left(\sigma_{j}^{\prime}\right) / \prod_{\sigma_{k} \neq \sigma_{j}^{\prime}}\left(\sigma_{k}-\sigma_{j}^{\prime}\right)$ and hence the sum of the residues of $I$ at the $\sigma_{1}^{\prime}, \cdots \sigma_{n}^{\prime}$ is equal to

$$
\sum_{\sigma_{j}^{\prime} i n \gamma_{1}}\left[\exp \left(t \sigma_{j}^{\prime}\right) / A_{j}\right]
$$

Hence by (18) the above expression tends to zero as $\varepsilon$ tends to zero and this ends the proof of the lemma. Now in order to finish the proof of the theorem we just write, 


$$
\left|Y_{l}(t, z \varepsilon)\right| \leqq\left|\sum_{\sigma_{j} i \gamma_{1}}\left[\exp \left(t \sigma_{j}\right) / A_{j}\right]+\cdots+\sum_{\sigma_{j}^{i n \gamma_{r}}} \exp \left(t \sigma_{j}\right) / A_{j}\right| .
$$

Using Lemma 1 one sees for all positive $t \leqq T$ that

$$
\left|Y_{l}(t, z, \varepsilon)\right| \rightarrow 0 \text { as } \varepsilon \rightarrow 0 \text {. }
$$

Now for proving the second part of the theorem, one notices from the preceding discussion that $Y_{l}(t, z, \varepsilon)=0(\varepsilon)$, i.e., there exist a number $M(z)$ such that $\left|Y_{l}(t, z, \varepsilon) / \varepsilon\right| \leqq M(z)$.

LEMMA A. Limit $_{\varepsilon \rightarrow 0}\left|E_{1,(i-1)} / A_{1}\right|=0$.

Proof. $\left|E_{1,(i-1)} / A_{1}\right|=\left|\sigma_{2} \cdots \sigma_{l-i+1}+\cdots+\sigma_{i+1} \cdots \sigma_{l-1} / j=2\left(\sigma_{j}-\sigma_{1}\right)\right|$ $(j=1, \cdots, l)$. Notice that as $\varepsilon$ tends to zero $\sigma_{1}$ tends to infinity while $\sigma_{j}(\varepsilon)$ tends to $\sigma_{j}(0)$ and hence this proves the lemma.

Lemma B. Limit ${ }_{\varepsilon \rightarrow 0}\left(E_{j(i-1)} / \sigma_{1}\right)={E^{\prime}{ }_{j,(i-1)}}$ where ${E^{\prime}{ }_{j,(i-1)}}$ is the coefficient of the $(i-1)$ th power of $\sigma_{j}(0)$ in the expression,

$$
\prod_{\substack{k \neq 2 \\ k \neq j}}^{l}\left[\sigma_{k}(0)-\sigma_{j}(0)\right](-1)^{j} \text {. }
$$

Proof. Notice that $E_{j,(i-1)}$ is the sum of the product of $\sigma$ taken $l-(i+1)$ at a time, i.e.,

$$
E_{j, i-1)}=\left[\sigma_{1} \cdots \sigma_{l-i+1}+\cdots+\sigma_{i+1} \cdots \sigma_{l-1}\right](-)^{j-1}(-1)^{i-1} .
$$

Therefore,

$$
E_{j,(i-1)} / \sigma_{1}=\sigma_{2} \cdots \sigma_{l-i+1}+\cdots+\left(\sigma_{i+1} \cdots \sigma_{l-1} / \sigma_{1}\right)(-1)^{i+j 2} .
$$

Now it is easy to see that $E_{j,(i-1)}$ tends to ${E^{\prime}{ }_{j,(i-1)}}$ as $\varepsilon$ tends to zero.

LEMMA C. Limit $_{\varepsilon \rightarrow 0}\left[E_{j,(i-1)} \exp \left(t \sigma_{j}\right) / A_{j}\right]=E^{\prime}{ }_{j,(i-1)} \exp (\sigma(0) t) / A_{j}^{\prime}$ $(j=2, \cdots)$ and $A_{3}^{\prime}=k=2\left[\sigma_{k}(0)-\sigma_{j}(0)\right]$.

Proof. Let us write.

$$
A_{j}=\left(\sigma_{1}-\sigma_{j}\right) \prod_{\substack{k=2 \\ k \neq j}}^{l}\left(\sigma_{k}-\sigma_{j}\right)=\sigma_{1} \prod_{\substack{k=2 \\ k \neq j}}^{l}\left(\sigma_{k}-\sigma_{j}\right)-\sigma_{j} \prod_{\substack{k=2 \\ k \neq j}}^{l}\left(\sigma_{k}-\sigma_{j}\right) .
$$

Therefore $A_{j} / \sigma_{1}$ tends to $A_{j}^{\prime}$ as $\varepsilon$ tends to zero. Now we use Lemma $\mathrm{B}$ and the proof of Lemma $\mathrm{C}$ will be completed.

$$
\text { Notice that } Y_{i}(t, z, 0)=\sum_{j=2}^{l} E^{\prime}{ }_{j,(i-1)} \exp \left(\sigma_{j}(0) t\right) / A^{\prime}{ }_{j}
$$

is the solution of the ordinary differential equation $L_{0}(D) Y=0$ with the initial conditions $\sigma_{t}^{k-1} Y_{i}(0, z, 0)=\delta_{j k} 1 \leqq j, k \leqq l-1$. Now we may 
sum the results of Lemmas $\mathrm{A}, \mathrm{B}$, and $\mathrm{C}$ in the following theorem.

THEOREM 6. Let $Y_{1}(t, z, \varepsilon), \cdots, Y_{l}(t, z, \varepsilon)$ be the solutions of the ordinary differential equation (7) with the initial conditions (8). Assume that; (1) $P_{l-1}(i z)$ is not identically zero. (2) assumption (0) on page (3). And finally (3) There exist numbers $M_{i}(z)$ and $\varepsilon_{0}$ such that for $0<\varepsilon \leqq \varepsilon_{0}$ and for almost all $z$ in $E^{m}$,

$$
\left|Y_{i}(t, z, \varepsilon)\right| \leqq M_{i}(z) \quad 1 \leqq i \leqq l, l>2 .
$$

Let $\varepsilon$ tend to zero on a sequence, then $Y_{i}(t, z, \varepsilon)$ tends to $Y_{i}(0, z, 0)$ for $0 \leqq t \leqq T, 1 \leqq i \leqq l-1$ and for almost all $z$ in $E^{m}$. Where $Y_{i}(t, z, 0)$ are the solution of the ordinary differential equation $L_{0}(D) Y=0$ with the initial conditions $\partial_{t}^{k-1} Y_{i}(0, z, 0)=\delta_{i k}, 1 \leqq i, k \leqq l-1$.

THeOREM 7. Assuming all the hypothesis of Theorem (5) then for each $z$ in $E^{m}$ we have,

$$
\begin{gathered}
\left|(1 / \varepsilon) Y_{l}(t, z, \varepsilon)-\left(1 / P_{l-1}(i z)\right) Y_{l-1}(t, z, \varepsilon)\right| \rightarrow 0 \\
\text { as } \varepsilon \rightarrow 0 \text { and for all } 0 \leqq t \leqq T .
\end{gathered}
$$

Proof.

$$
\begin{aligned}
& \left|\varepsilon^{-1} Y_{l}(t, z, \varepsilon)-P_{l-1}(i z)^{-1} Y_{l-1}(t, z, \varepsilon)\right| \\
& \quad \leqq\left|\left(\varepsilon A_{1}\right)^{-1} \exp \left(\sigma_{1} t\right)+\left(A_{1} P_{l-1}\right)^{-1} \exp \left(\sigma_{1} t\right) \sum_{2}^{l} \sigma_{i}\right| \\
& \quad+\mid \sum_{j=2}^{l}\left[\left(\varepsilon A_{j}\right)^{-1} \exp \left(\sigma_{j} t\right)+\left(P_{l-1} A_{j}\right)^{-1} \exp \left(\sigma_{j} t\right) \sum_{\substack{i=1 \\
i \neq j}}^{l} \sigma_{i} \mid .\right.
\end{aligned}
$$

It is clear that the first term of the above sum tends to zero as $\varepsilon$ tends to zero. Before dealing with the second term we shall reduce it into a simpler form. Notice that $\sum_{i=1, i \neq j} \sigma_{i}=\sum_{i=1} \sigma_{i}-\sigma_{j}=$ $-\varepsilon^{-1} P_{l-1}(i z)-\sigma_{j}$.

Then it is easy to see that

$$
\begin{gathered}
\sum_{j=2}^{l}\left[\left(\varepsilon A_{j}\right)^{-1} \exp \left(\sigma_{j} t\right)+\left(A_{j} P_{l-1}\right)^{-1} \exp \left(\sigma_{j} t\right) \sum_{\substack{i=1 \\
i \neq j}} \sigma_{i}\right] \\
=-\sum_{j=2}^{l}\left(A_{j} P_{l-1}\right)^{-1} \sigma_{i} \exp \left(\sigma_{j} t\right) .
\end{gathered}
$$

Now if, $\left|\sigma_{i}-\sigma_{j}\right| \geqq \delta>0(i, j=2, \cdots, l) i \neq j$ then it is clear that

$$
\left|\sum_{j=2}^{l}\left(A_{j} P_{l-1}\right)^{-1} \sigma_{j} \exp \left(\sigma_{j} t\right)\right| \rightarrow 0 \text { as } \varepsilon \rightarrow 0 \text {. }
$$

On the other hand, if for some $i$ and $i \neq j$ we have $\left|\sigma_{i}-\sigma_{j}\right|$ tends 
to zero as $\varepsilon$ tends to zero then we use the residue theorem to prove that

$$
\sum_{j=2}\left(A_{j} P_{l-1}\right)^{-1} \sigma_{j} \exp \left(\tau_{j} t\right) \rightarrow 0 \text { as } \varepsilon \rightarrow 0
$$

in the same way used before. The proof of Theorem 7 is ended.

Now we arrive at the main theorem of this paper.

THEOREM 8. Let the degree of $P_{j}(i z)$ be at most $k$ in $z(j=1, \cdots l)$ and assume that $P_{l-1}(i z)$ not identically zero. Denote by $u_{0}(t)$ the $l-1$ times $H_{p+k}$ continuously differentiable solution of the partial differential equation (4) for $\varepsilon=0$ in $0 \leqq t \leqq T$. If there exist two constants $\varepsilon_{0}>0$ and $C$ such that

$$
\begin{aligned}
& (19, \text { i }) \operatorname{Sup}_{z i n E}\left|Y_{j}(t, z, \varepsilon)\right| \leqq C \text { for } 0 \leqq t \leqq T \text { and } 0<\varepsilon \leqq 0 \quad(j=1, \cdots l) \\
& (19, \text { ii }) \operatorname{Sup}_{z i n E^{m}} \int_{0}^{T}\left|\varepsilon^{-1} Y_{l}(t, z, \varepsilon)\right| d t \leqq C \text { for } 0<\varepsilon \leqq \varepsilon_{0}
\end{aligned}
$$

where $y=Y_{j}$ the solutions of equation (7) with the initial conditions (8). Then equation (4) is an $H_{p}$-stable equation with respect to $u_{0}(t)$.

Proof. Let $u_{\varepsilon}(t, x)$ be $l$-times $H_{p+k}$ continuously differentiable solution of the partial differential equation (4) with the initial conditions (6). Then from Theorem 2 in [1] we may write

$$
\begin{aligned}
u_{\varepsilon}(t, x)=\sum_{j=1}^{l}(2 \pi)^{-m / 2} \int_{E^{m}} \int \exp (i x, z) Y_{j}(t, z, \varepsilon) \partial_{t}{ }^{j-1} \widehat{u}_{\varepsilon}(0, z) d z \\
\quad+2 \pi^{-m / 2} \int_{E^{m}} \exp (i x, z) \int_{0}^{t} \varepsilon^{-1} Y(t-\tau, z \varepsilon) \hat{f}_{\varepsilon}(\tau, z) d d z
\end{aligned}
$$

and

$$
\begin{aligned}
u_{0}(t, x)=\sum_{j=1}^{l-1}(2 \pi)^{-m / 2} \int_{E^{m}} \exp (i x, z) Y_{j}(t, z, 0) \partial_{t}^{j-1} \widehat{u}_{0}(0, z) d z \\
\quad+(2 \pi)^{-m / 2} \int_{E^{m}} \exp (i x, z) \int_{0}^{t} P_{l-1}{ }^{-1} Y_{l}(t-\tau, z) \hat{f}_{0}(\tau, z) d d z
\end{aligned}
$$

We have to prove that

$$
\left\|u_{\varepsilon}(t, x)-u_{0}(t, x)\right\|_{p} \rightarrow 0 \text { as } \varepsilon \rightarrow 0 .
$$

Let us write

$$
\begin{gathered}
\left\|u_{\varepsilon}(t, x)-u_{0}(t, x)\right\|_{p}=M(x)+N(x)+Q(x) \\
M(x)=\|(2 \pi)^{-m / 2} \sum_{j=1}^{l} \int_{E^{m}} \exp (i x, z) Y_{j}(t, z, \varepsilon) \partial_{t}^{j-1}\left[\widehat{u}_{\varepsilon}(0, z)-\widehat{u}_{0}(0, z)\right] \\
+(2 \pi)^{-m / 2} \int_{E^{m}} \exp (i x, z) Y_{l \partial t}^{l-1} \widehat{u}_{\varepsilon}(0, z) \|_{p}
\end{gathered}
$$




$$
\begin{aligned}
& N(x)=\int_{E^{m}}\left|\left(1+|z|^{2}\right)^{m / 2} \int_{0}^{t} \varepsilon^{-1} Y_{l}(t-\tau, z, \varepsilon) \hat{g}(\tau, z) d \tau\right|^{2} d z \\
& \times \hat{g}(\tau, z)=\hat{f}_{\varepsilon}(\tau, z)-\hat{f}_{0}(\tau, z) \\
& Q(x)=\int_{E^{m}}\left(1+|z|^{2}\right)^{m / 2} \int_{0}^{t}\left(\varepsilon^{-1} Y_{l}(t-\tau, z, \varepsilon)-P_{l-1}^{-1} Y_{l-1}(t-\tau, z, 0)\right) \\
& \times\left.\hat{f}_{0}(\tau, z) d \tau\right|^{2} d z \text {. }
\end{aligned}
$$

To prove the convergence of $M(x)$ we proceed as follows,

$$
\begin{gathered}
M(x) \leqq\left\|(2 \pi)^{-m / 2} \sum_{j=1}^{l-1} \int_{E^{m}} \exp (i x, z) Y_{j}(t, z, \varepsilon) \partial_{t}^{j-1}\left[\hat{u}_{\varepsilon}(0, z)-\widehat{u}_{0}(0, z)\right]\right\|_{p} \\
+\left\|(2 \pi)^{-m / 2} \int_{E^{m}} \exp (i x, z) Y_{l}(t, z, \varepsilon) \partial_{t}^{l-1} \widehat{u}_{\varepsilon}(0, z)\right\|_{p} .
\end{gathered}
$$

Using the condition $(19, \mathrm{i})$ and Ascoli's theorem we conclude

$$
M(x) \rightarrow 0 \text { as } \varepsilon \rightarrow 0 \text {. }
$$

Condition $(19$, ii) and Ascoli's theorem imply that

$$
N(x) \rightarrow 0 \text { as } \varepsilon \rightarrow 0 \text {. }
$$

For $Q(x)$ we proceed as follows. Notice that Theorem 6 shows that

$$
\left(1 / P_{l-1}(i z)\right) Y_{l-1}(t, z, \varepsilon) \rightarrow\left(1 / P_{l-1}(i z)\right) Y_{l-1}(t, z, 0)
$$

and Theorem 7 shows that

$$
\left|\varepsilon^{-1} Y_{l}(t, z, \varepsilon)-P_{l-1}^{-1} Y_{-1}(t, z, \varepsilon)\right| \rightarrow 0 \text { as } \varepsilon \rightarrow 0 .
$$

Therefore

$$
\left|\varepsilon^{-1} Y_{l}(t, z, \varepsilon)-P_{l-1}^{-1}(i z) Y_{l-1}(t, z, 0)\right| \rightarrow 0 \text { as } \varepsilon \rightarrow 0 .
$$

Consequently, using Ascoli's theorem once more we get

$$
Q(x) \rightarrow 0 \text { as } \varepsilon \rightarrow 0 \text {. }
$$

This ends the proof of Theorem 8 .

\section{REFERENCES}

1. Mitio Nagumo, On singular perturbation of linear partial differential with constant coefficients, Japan Academic Proc. 35 (1959), 449.

2. W. Wasow, Asymptotic expansion of O.D.E.

Received April 13, 1967, and in revised form September 27, 1968.

Fresno State College 



\section{PACIFIC JOURNAL OF MATHEMATICS}

\section{EDITORS}

H. ROYDEN

Stanford University

Stanford, California

Richard Pierce

University of Washington

Seattle, Washington 98105
J. DugundJI

Department of Mathematics

University of Southern California

Los Angeles, California 90007

BASIL GORDON

University of California

Los Angeles, California 90024

\section{ASSOCIATE EDITORS}

E. F. BECKENBACH

B. H. NeUmanN

F. WOLF

K. YOSHIDA

\section{SUPPORTING INSTITUTIONS}

UNIVERSITY OF BRITISH COLUMBIA

CALIFORNIA INSTITUTE OF TECHNOLOGY

UNIVERSITY OF CALIFORNIA

MONTANA STATE UNIVERSITY

UNIVERSITY OF NEVADA

NEW MEXICO STATE UNIVERSITY

OREGON STATE UNIVERSITY

UNIVERSITY OF OREGON

OSAKA UNIVERSITY

UNIVERSITY OF SOUTHERN CALIFORNIA
STANFORD UNIVERSITY

UNIVERSITY OF TOKYO

UNIVERSITY OF UTAH

WASHINGTON STATE UNIVERSITY

UNIVERSITY OF WASHINGTON

$* * \quad * \quad *$
AMERICAN MATHEMATICAL SOCIETY
CHEVRON RESEARCH CORPORATION
TRW SYSTEMS
NAVAL WEAPONS CENTER




\section{Pacific Journal of Mathematics}

Vol. 30, No. $1 \quad$ September, 1969

William Wells Adams, Simultaneous diophantine approximations and cubic irrationals ..................................... 1

Heinz Bauer and Herbert Stanley Bear, Jr., The part metric in convex

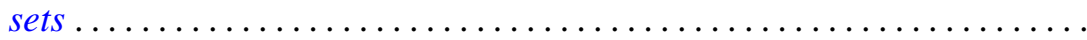

L. Carlitz, A note on exponential sums ...................... 35

Vasily Cateforis, On regular self-injective rings ................. 39

Franz Harpain and Maurice Sion, A representation theorem for measures on infinite dimensional spaces ......................... 47

Richard Earl Hodel, Sum theorems for topological spaces .............. 59

Carl Groos Jockusch, Jr. and Thomas Graham McLaughlin, Countable

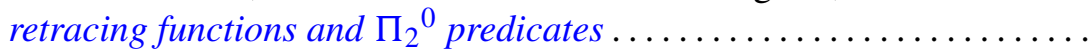

Bjarni Jónsson and George Stephen Monk, Representations of primary

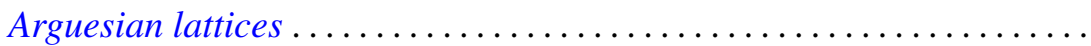

Virginia E. Walsh Knight, A continuous partial order for Peano continua...................................... 141

Kjeld Laursen, Ideal structure in generalized group algebras ........... 155

G. S. Monk, Desargues' law and the representation of primary lattices . . . 175

Hussain Sayid Nur, Singular perturbation of linear partial differential equation with constant coefficients ..........................

Richard Paul Osborne and J. L. Stern, Covering manifolds with cells ... 201

Keith Lowell Phillips and Mitchell Herbert Taibleson, Singular integrals in several variables over a local field...

James Reaves Smith, Local domains with topologically $T$-nilpotent radical....

Donald Platte Squier, Elliptic differential equations with discontinuous coefficients .................................

Tae-il Suh, Algebras formed by the Zorn vector matrix...

Earl J. Taft, Ideals in admissible algebras . .................... 259

Jun Tomiyama, On the tensor products of von Neumann algebras........ 263

David Bertram Wales, Uniqueness of the graph of a rank three group ..... 271

Charles Robert Warner and Robert James Whitley, A characterization of regular maximal ideals ......................... 